\title{
Reconstruyendo Espacios Urbanos en Santiago y Concepción: Una Lectura desde la Geografía Social Contemporánea
}

\author{
Hugo Marcelo Zunino Edelsberg, Ph.D. \\ Universidad de Chile \\ Departamento de Geografía \\ Portugal 84, Santiago, Chile \\ Email: zunino1@uchile.cl
}

\begin{abstract}
Resumen
Tomando como casos de estudio dos proyectos urbanos ejecutándose en las ciudades de Santiago y Concepción (Chile), este artículo examina las estructuras de poder que hacen posible estos emprendimientos. Para ello, se desarrolla y aplica un referente metodológico que pretende reconocer tanto las influencias estructurales como la capacidad de los individuos para forjar su propio destino. Se concluye reflexionando, someramente, sobre los desafíos de los geógrafos interesados en temáticas urbanas y sociales.
\end{abstract}

\begin{abstract}
Taking two urban redevelopment projects taking place in the cities of Santiago and Concepción (Chile), this article examines the power relations sustaining these initiatives. In this vein, a methodological framework is suggested and applied to consider both, structural forms of constraints and the capacity of individuals to make a difference in their own destiny. In the concluding section, some preliminary thoughts are layout regarding the challenges for geographers interested in urban and social issues.
\end{abstract}

\section{Introducción}

La literatura geográfica especializada en temas urbanos en Chile y Latinoamericana le ha otorgado, insistentemente, un fuerte peso analítico a las condiciones macro-estructurales que están re-dibujando el tejido morfológico, social y económico de los conglomerados urbanos (para el caso Chileno véase, entre otros, DE MATTOS 1999, 2003; HIDALGO 2004; ORTIZ y ESCOLANO 2005; ORTIZ y SCHIAPACASSE 1998). Así, diversas «patologías urbanas» de la ciudad capital de Chile como la concentración demográfica y productiva, la contaminación atmosférica, la fragmentación urbana, la exclusión social, y la segregación social se han relacionado con la consolidación de un régimen neoliberal nacido bajo el alero del Régimen Militar y profundizado bajo los gobiernos de la Concertación de Partidos por la Democracia (1990 - 2006). Por cierto, las «patologías urbanas» han existido a lo largo de la historia, pero éstas han adquirido una nueva dinámica en las últimas décadas. En particular, bajo la administración Lagos (2000-2006) y ante la necesidad de darle continuidad al modelo económico vigente, el Estado-nacional ha ganado peso a través de las intervenciones urbanas dirigidas por el Ministerio de la vivienda y Urbanismo y, con particular fuerza, por el Ministerio de Obras Públicas.

Si bien la línea de investigación que le otorga un fuerte peso a la relación entre los procesos que se desenvuelven en la esfera macro-sociológica (a nivel de las estructuras sociales) con los cambios en la forma, organización y funcionalidad de las ciudades ha aportado significativamente al quehacer geográfico, existe una amplia gama de temas que permanecen al margen de los cuestionamientos de esta línea de razonamiento. Un ejemplo de este vacío 
intelectual es la falta de penetración analítica sobre cuáles son las relaciones de poder que se dan entre los agentes urbanos involucrados en la construcción de la ciudad (ZUNINO 2001, 2002). No obstante que esta línea de investigación es crucial para detectar nodos estratégicos en donde el poder es ejercido para alcanzar fines predeterminados y como, eventualmente, se pueden desestabilizar las relaciones de poder existentes, sólo existen evidencias parcializadas y nociones mecánicamente aceptadas sobre la creciente influencia del capital privado en la construcción social de la ciudad. Así, en Chile, no existen estudios sistemáticos sobre los mecanismos y las estrategias empleadas para llevar adelante emprendimientos urbanos lo que, como cualquier iniciativa, conllevan ganadores y perdedores.

El objetivo principal de esta comunicación es examinar, con cierto nivel de detalle, las relaciones de poder que están sosteniendo dos proyectos emblemáticos que tienen el potencial de afectar la funcionalidad y organización de las ciudades: el Proyecto Portal Bicentenario (PPB) en Santiago y el Proyecto Ribera Norte (PRN) en Concepción. A diferencia de posiciones neo-estructuralistas cuya aproximación a la investigación es deductiva, aquí se propone una aproximación inductiva, bajo la premisa que son acontecimientos que se desenvuelven en la escala «local» los puntos críticos en donde se le otorga forma concreta a procesos que son gatillados por procesos nacionales o mundiales.

Este enfoque no es contrario ni desmerece los enfoques «neo-estructuralista», los cuales son capaces de descifrar el contexto general de oportunidades y restricciones para el accionar de los agentes urbanos; el punto de contención es que el ser humano retiene no siempre y dependiendo de las circunstancias - la capacidad de resistir, contra-argumentar y provocar cambios no-esperados a los dictámenes provenientes de las esferas nacionales y supranacionales. 1 Esta comunicación busca conciliar metódicamente lo deductivo e inductivo, lo general y lo local, la estructura y la agencia del ser humano (el actor situado como veremos más adelante).

\section{Marco Teórico - Conceptual}

El sustento teórico-metodológico de esta investigación está desarrollado en ZUNINO 2002) y su aplicación empírica queda reflejada en dos casos de estudio analizados por el mismo autor $(2005,2006)$. A pesar que estos textos son necesarios para una comprensión acabada de lo que aquí se expone, a continuación se ofrece un breve resumen teórico-conceptual el cual tiene como fin encauzar al lector hacia los aspectos fundamentales de esta comunicación. Asimismo, se avanza en ofrecer una metodología comparativa para analizar cambios urbanos, bajo la premisa que para comprender la construcción social del territorio no basta con una mirada genérica a procesos estructurales, sino es necesario llegar hasta el detalle mismo de cómo se ejerce el poder y a las tecnologías de gobierno desplegadas para controlar el espacio y a la sociedad (la microfísica del poder, empleando un silogismo Foucaultiano). Esta aproximación permite detectar fisuras en el sistema de toma de decisiones y avanzar hacia una planificacion urbana más participativa e inclusiva.

Cómo punto de partida teórico-conceptual se cuestionará qué se entiende por «estructura social» y por «agente urbano». Para ello, ocuparemos referentes teoricos provenientes de distintas disciplinas incluyendo la geografía, la sociología, y la ciencia política. Siguiendo a GIDDENS $(1979,1984)$ las estructuras sociales pueden ser concebidas como reglas sociales formales (escritas) e informales (no escritas) que conforman un enjambre de prescripciones que «estructuran» el accionar del agente individual (en este caso, se hablará, simplemente, del agente urbano). Desde un punto de vista práctico, varios puntos aparecen conflictivos para llevar a la práctica estas disquisiciones teóricas. Primero, GIDDENS no ofrece herramientas metodológicas y una terminología adecuada 
para interpretar las reglas sociales operando en un espacio-tiempo determinado. Así, la noción de «reglas» permanece en un limbo analítico. Segundo, las reglas sociales, como creaciones humanas, están sujetas a cambios, lo cual abre espacios para modificar el funcionamiento del sistema social. GIDDENS permanece silencioso al respecto. Tercero, paradojicamente, GIDDENS no desarrolla con la profundidad necesaria el concepto de «poder», el cual permanece anclado en sujetos que tienen a su disposición ciertos recursos que les permiten tomar decisiones. Bajo esta óptica el poder es conceptualizado como algo que se "posee» y es parte de estructuras de dominación y representación preexistentes, debilitando su propia concepción de actor situado, condicionado pero no determinado por macro-estructuras de dominación.

Para sortear las dificultades metodológicas que conlleva llevar las disquisiciones teóricas de GIDDENS al ámbito de la geografía aplicada y utilizarlas para descifrar las relaciones de poder imbricadas en la construcción social del territorio, se propone seguir los siguientes movimientos analíticos. Primero, tomar una aproximación Foucaultiana a la noción de poder (ver FOUCAULT 1979, 1980, 1991, 1995), lo cual significa, en lo medular, investigar como el poder es ejercido para alcanzar fines determinados, lo cual se plasma conceptualmente en descifrar las «tecnologías de gobierno» empleadas por distintos actores operando tanto en el ámbito público como privado. Segundo, poner especial atención en el carácter «vertical» de la toma de decisiones, lo cual se relaciona con las acciones y decisiones de actores que operan en distintas escalas funcionales: nacional, regional, y local.

Cabe apuntar que la sola definición de estas categorías es ya un constructo metodológicodiscursivo pues las escalas geográficas no están dadas ni son fácilmente definibles, como se ha discutido acaloradamente en años recientes en la literatura anglosajona (véase, por ejemplo, BRENNER, 2001, MACLEOD y GOODWIN 1999). Hay que tener en cuen- ta también que ciertos actores sociales operan en distintas escalas geográficas, lo que les permite tener un particular alcance a la hora de hacer prevalecer ciertos intereses. Tercero, utilizar la categoría de reglas sociales propuestas por OSTROM (1986) en orden a descifrar los canales por medio de los cuales se ejerce el poder (ver también WATERSTONE 1995, 1996). Estas reglas sociales permiten interpretar como se reproduce continuamente una estructura social y cuáles son los canales utilizados para el ejercicio del poder.

El conjunto de reglas sociales operando a distintas escalas funcionales permiten esbozar la transitoriedad y fragilidad de las estructuras sociales responsables de la construcción, reproducción y transformación de los artefactos urbanos que componen la ciudad $\mathrm{y}$ hacen posible su funcionalidad.

\section{Metodología}

La definición de actores estratégicos constituyó la primera fase de esta investigación. Estos fueron definidos mediante las reglas de posición, alcance y autoridad especificadas por OSTROM (1986). Un actor estratégico queda definido por ocupar un sitial en un órgano de toma de decisiones (una posición) y por tener una voz y la capacidad para decidir sobre aspectos fundamentales de proyectos urbanos específicos. Estos actores estratégicos incluyeron, para el caso del Proyecto Portal Bicentenario en Santiago, al Gerente General, a técnicos y profesionales del Ministerio de Vivienda, profesionales de la Intendencia Regional de la Región Metropolitana, asesores directos del Alcalde de Cerrillos, Presidentes de Juntas de Vecinos y gente que habita en la vecindad del proyecto y que pueden verse negativa o positivamente afectados. Para el caso del Proyecto Ribera Norte en Concepción, se consideró al Coordinador de la «Oficina Ribera Norte» (ORN), al jefe de la Unidad Técnica de la ORN, al jefe de la unidad social de la ORN, a personal técnico de la Municipalidad e Intendencia Regional del Bio-Bio, a la comunidad organizada en «Comités de Defensa», a los 
Presidentes de Juntas de Vecinos y a la población afectada.

Posteriormente, se definió el nivel funcional en que cada actor operaba. Para esto se consideró el nivel de coordinación (nivel en donde confluyen actores con la capacidad de influir en la directrices emanadas de las autoridades nacionales) y el nivel operacional (nivel en donde los actores sociales locales materializan, validan o legitiman el proyecto). Una vez identificado el nivel funcional en que cada actor operaba, entre los años 2002 y 2004 se llevaron una serie de 32 entrevistas semi-estructuradas con actores estratégicos y entrevistas abiertas con dirigentes vecinales y pobladores.

La pauta para las entrevistas se fundamentó en la categorización de regla sociales propuestas por OSTROM (reglas de posición, borde, autoridad, alcance, información, y distribución de costos y beneficio). A modo ilustrativo señalaremos algunas preguntas directrices: ¿Cuál es su función dentro del proyecto? (reglas de posición y alcance) ¿Qué elementos del proyecto es usted capaz de afectar? (reglas de autoridad y alcance) ¿Quién produce la información utilizada en la toma de decisiones? ¿Cómo se distribuye dicha información? (reglas de información) ¿Quiénes ganan, quiénes pierden con la materialización del proyecto? (reglas de distribucion). Cada entrevista fue grabada y luego transcrita textualmente para su posterior análisis.

Descifrar la compleja red de reglas sociales operando permitió identificar las estructuras que le dan sustento a los proyectos y las estrategias y tácticas seguidas (o no) por la comunidad afectada para resistir los planes tecnocráticos elaborados para re-desarrollar los espacios urbanos involucrados. Esto resulta consistente con la aproximación de FOUCAULT $(1991,1995)$ quien sugiere que todo el andamiaje para ejercer el poder va a la par con la existencia de distintos contradiscursos y modos de resistencia; lo cual sugiere - a diferencian de aproximaciones neoestructuralistas - que el poder no emana de una «super-estructura» que gatilla mecánicamente cambios en la morfología urbana: la construccion social de la ciudad es, en definitiva, una obra humana, de individuos que están defendiendo intereses y agendas particulares. Por cierto que las estructuras sociales son limitantes, pero, siguiendo a GIDDENS (1984), éstas también posibilitan el accionar de una gama de individuos. Así, el poder es siempre ejercido al mismo tiempo desde «arriba hacia abajo» y desde «abajo hacia arriba». FOUCAULT denomina a esto la capilaridad de las relaciones de poder (1980: 201).

Una salvedad está en orden. El esbozo metódico aquí presentado no tiene la intención de realizar una lectura «objetiva» o "positivista» de las relaciones de poder que le están dando expresión concreta a ambos proyectos. No se pretende llegar más allá de una interpretación fundamentada del investigador sobre las diversas y complejas relaciones sociales que se dan en un espacio-tiempo determinado. Con todo, la rigurosidad metodológica seguida permite detectar aquellos nodos críticos en donde las relaciones de poder son establecidas por las cúpulas en posiciones de privilegio y las posibles acciones de resistencia para hacer frente a la construcción autoritaria del espacio urbano (véase Zunino 2006). Así, este constructo metodologico pretende tener validez en el campo aplicado; específicamente detectando cómo el poder es ejercido, que coyunturas son aprovechadas o aprovechables por ciertos actores y cómo, eventualmente, el desarrollo urbano puede tornarse más equitativo y democrático.

\section{Resultados}

El Proyecto Portal Bicentenario tiene su origen en el afán de posicionar discursivamente a ciertos actores en la cúspide del poder político. Este proyecto pretende re-desarrollar el espacio ocupado por el ex aeródromo Los Cerrillos (unas 250 hectáreas), ofreciendo oportunidades para emprender negocios inmobiliarios dirigidos a estratos medios y medio-altos, junto con exaltar valores cívi- 
cos y comunitarios. Llamó la atención que numerosos informantes que ocupaban un papel técnico en la Intendencia Metropolitana y en el Ministerio de Vivienda y Urbanismo dudaron de la viabilidad técnica del proyecto, siendo recurrente la afirmación: «aquí lo único que cabe es construir viviendas sociales».

El proyecto en comento se esta ejecutando bajo un fuerte comando centralizado, personificado por un Gerente General. Esta posición mantenía estrechos lazos con el entonces Ministro de Vivienda y Urbanismo y controlaba pequeños pero influyentes equipos técnicos que operaban en distintas reparticiones del Estado Nacional (regla de alcance). El Gerente General junto a sus asesores más directos tenía la facultad de decidir que tipo de estudios realizar y como distribuir la información (reglas de información). Para conseguir la realización rápida y expedita del PPB una de las estrategias de gobierno empleada fue elevar a la municipalidad desde el nivel operacional - en donde podía ejercer a plenitud la regla de autoridad referida a la elaboración de planes de uso del suelo - hacia el nivel de coordinación o implementación, en el que dominaban discusiones técnico-políticas y la municipalidad se encontraba con escasas posibilidades de influir en la toma de decisiones debido a la falta de recursos financieros y técnicos.

Una instancia central para mantener funcionando el aparataje de poder que pretendía reconfigurar espacial y socialmente a la $\mathrm{Mu}-$ nicipalidad de Cerrillos eran los «comités consultivos», los cuales se reunían perdiódicamente para tratar algún problema particular. Un funcionario técnico de la Intendencia Metropolitana reflexiona sobre los roles de estos comités:

[El Gerente General]....viene aquí y le pide la opinión a otras personas que en vez de redactar los términos de referencia sólo los leen....y como tiene hartos amigos ya tiene un respaldo y le puede decir al Ministro: revisamos los términos y lo hicimos entre todos.
Este fragmento nos indica que los comités técnicos funcionaban principalmente con el objeto de legitimar el proyecto y evitar que surgiesen conflictos entre las instituciones con intereses en la materialización del PPB. Asimismo, era una instancia en donde la municipalidad tenía una voz, pero no el voto (regla a agregación) para imponer su propia visión.

La participación ciudadana se redujo durante el periodo analizado a la distribución de folletería elemental y prácticamente ningún vecino entrevistado pudo articular una opinión que fuese más allá de lo contenido en los folletos e informaciones de prensa, denotando que la comunidad potencialmente afectada por el proyecto se mantenía al margen de las decisiones fundamentales.

En el caso del Proyecto Ribera Norte (PRN), la autoridad nacional en la década de los noventa intentó la pronta erradicación de los pobladores que ocupaban ilegalmente un sitio cercano al centro de la ciudad y de alta plusvalía. Con ese fin se formó la organización de carácter informal denominada Oficina Proyecto Ribera Norte, compuesta, en lo medular, por un Coordinador (nivel de coordinación) y dos unidades que desplegaban su accionar en el nivel operacional: la Unidad Técnica y la Unidad Social. El Coordinador dependía directamente del Ministro de Vivienda y Urbanismo.

A diferencias de la estructura de relaciones comandando el PPB, en el caso del PRN la Municipalidad de Concepción, actuando en el nivel operacional, tuvo una participación activa y generó su propia información basada en entrevistas y encuestas directas con la población involucrada. Esto le permitió al municipio confrontar las ideas provenientes de ORN desde una posición de fortaleza. Unamos a esto la activa participación de los pobladores, quienes no sólo se dieron una estructura interna compleja y altamente funcional, sino fueron capaces también de movilizarse, y, en definitiva, evitar la erradicación hacia sectores periféricos del Gran Concepción. 
La tecnología de gobierno fundamental empleada por la ORN fue dividir drásticamente los canales de negociación y acuerdo: por una parte la Unidad Técnica trabajaba con la Municipalidad y el sector privado una estrategia para maximizar la inversión inmobiliaria, mientras que la Unidad Social y la Municipalidad de Concepción tenían a su cargo la relación con los pobladores. Cabe destacar la fuerte incidencia que tuvo la Municipalidad en la puesta en marcha del PRN, actuando tanto en el ámbito técnico como social. La existencia de dos canales separados de negociación, sin embargo, fue funcional a los intereses de ORN pues le permitió controlar el flujo de la información y mantener dos canales autónomos y paralelos de negociación y compromiso. Era solamente a nivel de la coordinación del proyecto en donde las piezas de negociación se juntaban y daban origen a una decisión basada en una regla informal de distribución salomónica: ni los inversionistas ganarán todo, ni los pobladores ganarán todo. De hecho, los pobladores a pesar de ser erradicados de un lugar de alta plusvalía, fueron capaces de permanecer en un espacio vecino, a tan solo unos dos kilómetros de la toma ilegal desde donde fueron desplazados.

Aunque no se puede hablar de una victoria del «movimiento poblacional», si fueron capaces de mantener una buena localización dentro de la ciudad, con excelentes niveles de conectividad con el Centro de Negocios de Concepción, y lograron soluciones habitacionales con estándares de calidad muy superiores a las soluciones habitacionales que se dan en otros lugares de nuestro país (para una visión detallada de este proyecto véase ZUNINO, 2005).

\section{Discusión}

HARVEY (ver, por ejemplo, 1982, 1989) y otros geógrafos trabajando desde la vertiente neo-estructuralista tienden a ver el proceso de desarrollo urbano-regional como homogenizante, bajo la premisa - no siempre explícita - que el sistema capitalista contemporáneo impacta mecánicamente la morfología urbana y en las relaciones de poder entre los distintos actores involucrados en programas de desarrollo urbano y proyectos específicos. Estos proyectos localizados tienen relevancia pues afectan de manera directa la funcionalidad de las ciudades.

Complementando ecléctica pero consistentemente los aportes de GIDDENS, FOUCAULT y OSTROM se hace posible entender la construcción social de las ciudades como un proceso eminentemente político, en el cual las macro-estructuras (e.g., el modelo neoliberal Chileno) no son determinantes y cabe el espacio para desarrollar contra-discursos y ejercer influencia en cómo las ciudades son construidas por seres humanos defendiendo agendas particulares.

El Caso del PRP en Concepción es emblemático en el sentido que a pesar de la fuerte presión ejercida por el sector inmobiliario para abrir a la inversión privada todo el sector de la ribera norte del río Bio-Bio no lograron su fin al verse confrontados por grupos activos y movilizados de pobladores que contaban con el respaldo sutil pero influyente de la Municipalidad de Concepción. Esta situación contrasta, fuertemente, con el autoritarismo que imperó en el Proyecto Portal Bicentenario, en donde un grupo de agentes dotados de reglas informales de autoridad y alcance, comandaron el proceso urbano, relegando, estratégicamente, a la comunidad afectada y a la Municipalidad de Cerrillos. Por cierto no se puede caer en una visión determinista/simplista, tanto la municipalidad como la población afectada tenían las facultades para contrarrestar la influencia de actores dotados de ciertos recursos para ejercer el poder.

Siguiendo esta lógica y el trabajo de GIDDENS, podemos afirmar que los actores sociales no están condicionados, predeterminados por macro estructuras actuando mecánicamente sobre ellos; tampoco estamos en presencia de actores sociales «libres» pues ellos están necesariamente condicionados (pero no determinados) por las estructuras reproducidas a lo largo del tiempo y espacio. Es por tanto, analíticamente útil 
conceptualizar al ser humano como un «actor situado» o «actor estructurado»; esto es un individuo que opera bajo las restricciones y posibilidades que le ofrece un sistema social dado. Como corolario a esta aproximación conceptual, se puede comprender que cuando existe el ejercicio del poder están siempre abiertos los espacios para el cambio, entendiendo por ello la capacidad de los actores situados para desafiar los discursos predominantes, enriquecerlos y, eventualmente, avanzar hacia formas más democráticas e inclusivas de desarrollo urbano.

Si se analizan las reglas formales vigentes en materia urbana incluyendo las normativas ZODUC, ADUC y la «gran reforma urbana» promovidas por el Ministerio de la Vivienda y Urbanismo, es dable llegar a la conclusión que el desarrollo de las ciudades Chilenas está siendo comandado por los inversionistas privados. El caso en Concepción nos muestra, sin embargo, que existen espacios de resistencia que pueden ser aprovechados: detectando las fisuras del sistema y actuando «con las armas del maestro». Esta última afirmación no significa, en absoluto, una servilismo; muy por el contrario, señala rumbos para cambiar el sistema de relaciones de poder «desde adentro hacia fuera». Como sugiere FOUCAULT (1995: 92), en la historia se le ha dado excesiva importancia a los movimientos desde arriba (a los grandes cambios macro-estructurales), perdiendo de vista las formas de resistencia localizadas que pueden, eventualmente, congregarse en movimientos más amplios que, en forma paralela a cambios en la esfera macro-sociológica, pueden sentar las bases para cambios sociales de magnitud $i$ No es cierto, acaso, que las grandes revoluciones (de cualquier signo) tuvieron su punto de partida en nodos de resistencia delimitados espacial y temporalmente?

\section{CONCLUSIONES}

Además de ofrecer un resumen sumario de lo expuesto y de los resultados alcanzados, esta sección final se centrará en reflexionar sobre el quehacer geográfico. El punto de partida de esta comunicación fue comprender que la construcción, reproducción, y destrucción de los artefactos urbanos que articulan y le dan funcionalidad a la ciudad no son obras de factores puramente externos; son procesos guiados por «actores situados» los cuales actúan bajo las restricciones y posibilidades que ofrece una estructura social dada, la cual fue definida aquí como un conjunto de reglas formales e informales actuando en distintas escalas funcionales.

El análisis de ambos proyectos indica la relevancia de desarrollar estudios comparativos sobre la construcción social de las ciudades de modo de precisar los mecanismos utilizados y las potencialidades para avanzar hacia formas más inclusivas y democráticas de hacer ciudad. Una de las estrategias discursivas de gobierno más utilizadas es convencer a los estudiosos del tema que «no hay alternativa»; aquí se plantea que si las hay.

Desde el particular punto de vista del autor, la geografía social contemporánea debiese avanzar desde estudios meramente descriptivos - los cuales por cierto son necesarios y representan una tarea fundamental para los geógrafos - hacia el análisis de las relaciones sociales que están constituyendo el territorio; permitiendo, de esta manera, desarrollar una disciplina más comprometida con el desarrollo nacional. No se trata sólo de constatar sino, por sobre todo, ofrecer alternativas. En los dos casos de estudio que someramente tocó esta investigación se pueden ofrecer recomendaciones político-territoriales para avanzar hacia formas más inclusivas de desarrollo urbano.

En el caso del Proyecto Portal Bicentenario en Santiago, el simple hecho de dilucidar la construcción autoritaria de un territorio dado puede gatillar cambios en la forma en que la municipalidad enfrenta el tema haciendo uso de las reglas formales de autoridad que posee, por ejemplo, aquella referida a la elaboración de planes de uso de suelo. En el caso del Proyecto Ribera Norte en Concepción, para afianzar formas democráticas de hacer 
ciudad parece imperioso romper con la autoconstruida separación entre los intereses de los inversionistas privados y los pobladores, pues genera dos flujos separados de información y condiciona el modo en que se toman las decisiones (regla de agregación) queda a merced del coordinador del proyecto.

Una geografía moderna y comprometida con los problemas que afectan al país implica establecer un diálogo fluido y de igual a igual con otras disciplinas, rompiendo la separación artificial entre las distintas disciplinas $\mathrm{y}$, junto con los métodos cuantitativos de análisis, darle la debida importancia a metodologías cualitativas de investigación. En resumen, resulta imperiosa una renovación conceptual, metodológica y teórica, tomando como referente las experiencias de países en donde el quehacer geográfico goza de alta incidencia en la toma de decisiones en distintos campos. Más que meros descriptores de la realidad que nos circunda, los geógrafos deben reconocerse como seres políticos cuya labor debiese estar orientada hacia la resolución de los grandes temas nacionales.

\section{REFERENCIAS}

BRENNER, N., 2000. The limits of scale? Methodological reflections on scalar structuration. Progress in Human Geography, 24(2): $361-378$

DE MATTOS, C. A., 1999. Santiago de Chile, globalización y expansión metropolitana: lo que existía sigue existiendo, Eure, 25(76): 29-56.

DE MATTOS, C. A., 2003. Globalización y transformación metropolitana en el caso de Santiago. En: F. ARENAS, R. HIDALGO y J. L. COLL (Eds.) Los nuevos modos de gestión de la metropolización, pp. 27-56. Pontificia Universidad Católica de Chile: Santiago, Chile.

FOUCAULT, M., 1979. The history of sexuality. Volume 1: an introduction. Guildford: Londres.
FOUCAULT, M., 1980. Power and Strategies. En: C. GORDON (Ed.) Power / Knowledge: Selected interviews and other writings, pp. 134-145. Pantheon Books: New York.

FOUCAULT, M., 1982. The subject and power, en: H. L. DREYFUS y P. RABINOW (Eds,) Michel Foucault, beyond structuralism and hermeneutics, pp. 208-226. University of Chicago Press: Chicago, ILL.

FOUCAULT, M., 1995. Discipline and Punish. The birth of the prison. Vintage Books: Nueva York.

HARVEY, D., 1982. The limits to capital. Chicago; Oxford: University of Chicago Press: Chicago, ILL

HARVEY, D., 1989. The condition of postmodernity: an enquiry into the origins of cultural change. Blackwell: Oxford, England; New York, NY.

HIDALGO, R., 2004. De los pequeños condominios a la ciudad vallada: las urbanizaciones cerradas y la nueva geografía social en Santiago de Chile. Eure, 30(91): 29-52.

ORTIZ, J. y S. ESCOLANO, 2005. Crecimiento periférico del Gran Santiago. ¿Hacia la desconcentración funcional de la ciudad?. En: Coloquio Internacional de Geocrítica. Los agentes urbanos y las políticas sobre la ciudad. Santiago: Instituto de Geografía Pontificia Universidad Católica de Chile de Chile y Departamento de Geografía Humana Universidad de Barcelona, 24-27 de mayo 2005. Inédito

ORTIZ, J. y P. SCHIAPACASSE, 1998. Dimensiones latentes de la diferenciación del espacio social en una metrópolis latinoamericana. El caso del Gran Santiago. Geographicalia, 36:111-130.

OSTROM, E., 1986. An agenda for the study of institutions. Public Choice, 48: 3-24.

GIDDENS, A., 1979. Central problems in 
social theory: action, structure and contradiction in social analysis. Macmillan: Londres

GIDDENS, A., 1984. The constitution of society: outline of the theory of structuration. Polity Press: Cambridge

MACLEOD, G. y GOODWIN, M., 1999. Space, scale and state strategy: rethinking urban and regional governance, Progress in Human Geography, 23(4): 503-527

WATERSTONE, M., 1995. Social theory, structuration and institutional analysis: grounding theory and theorizing practice. Trabajo leído en el Departamento de Geografía, Universidad de Oregon. Inédito.

WATERSTONE, M., (1996) A conceptual framework for institutional analysis of transboundary water resource management: theoretical perspectives. In: J. GANOULIS, L. DUCKSTEIN, P. LITERATHY and I. BOGARDI (Eds) Transboundary water resource management. Institutional and engineering approaches, pp. 9-18. Springer: New York

ZUNINO, H., 2001. La planificación urbana en el Chile de hoy: 'actores sociales estructurados' y la construcción de redes de gobernabilidad. Revista de Geografía Norte Grande, 28:73-78.

ZUNINO, H., 2002. Formación institucional y poder: investigando la construcción social de la ciudad. EURE, 28(84):103-116.

ZUNINO, H., 2005. Social theory at work: Analyzing multi-level power relations in the redevelopment of Concepcion's riverfront. Cidades, 2(4): 315-337

ZUNINO, H. 2006. Power relations in urban decision making: neoliberalism, «technopoliticians», and authoritarian redevelopment in Santiago, Chile. Urban Studies, 43(10). En prensa

1 El silogismo «neo-estructuralista» se emplea en esta comunicación de manera genérica, indicando una aproximación al hecho urbano que pone el acento analítico en los cambios gatillados por modificaciones en el funcionamiento del sistema económico capitalista, particularmente la así llamada transición desde el capitalismo Fordista al régimen Post-Fordista de acumulación el cual se ha asociado al advenimiento del «neo-liberalismo» como paradigma económico y de gestión predominante. 